\title{
Measuring food provision in Western Australian long day care (LDC) services: a weighed food record method/protocol at a service level
}

\author{
Ros Sambell ${ }^{*} \mathbb{D}$, Ruth Wallace ${ }^{\dagger}$, Leesa Costello ${ }^{\dagger}$, Johnny Lo $^{\dagger}$ and Amanda Devine ${ }^{\dagger}$
}

\begin{abstract}
Background: There are currently 1.3 million children utilising Early Childhood Education and Care (ECEC) services in Australia. Long day care (LDC), family day care and out of school hours care currently provide this service in different environments. This research reports findings from a LDC perspective. Children can consume 40-67\% of their food intake whilst at LDC services, this highlights the importance of monitoring food provision at a service level. There are several methods to measure food provision which typically focus on intake at an individual level. There is limited evidence of measuring food provision accurately at a service level and for young children. Accurate and consistent dietary assessment methods are required to determine compliance with dietary guidelines and to provide rigour for comparison between studies.
\end{abstract}

Methods: Convenience sampling was used to recruit 30 LDC services and food provision assessed over two consecutive days. To ensure consistency, trained researchers weighed raw food ingredients used in food preparation at each service. Food and food weights were allocated to food groups to determine average serves of food group provision at morning tea, lunch and afternoon tea per child. All data were entered into Foodworks for dietary analysis and compliance with dietary guidelines was assessed using Wilcoxon signed-rank and one-sample t-tests (SPSS).

Discussion: This paper outlines the process of data collection for the measurement and auditing of food provision and food waste at a service level. There is an urgent need to ensure that food provision at a service level complies with current dietary guidelines and is accurately assessed. Following a standard method of data collection will allow a more accurate comparison between studies and allow change to be monitored more accurately over time to guide decision makers.

Trial registration: As this research project is conducted at a service level and not a clinical trial, registration was not required.

Keywords: Long daycare, Food provision, Measurement, Study protocol, Childcare, Nutrition

\footnotetext{
* Correspondence: r.sambell@ecu.edu.au

${ }^{\dagger}$ Ros Sambell, Ruth Wallace, Leesa Costello, Johnny Lo and Amanda Devine

contributed equally to this work.

Edith Cowan University, 270 Joondalup Drive, Joondalup, WA 6027, Australia
}

(c) The Author(s). 2019 Open Access This article is distributed under the terms of the Creative Commons Attribution 4.0 International License (http://creativecommons.org/licenses/by/4.0/), which permits unrestricted use, distribution, and reproduction in any medium, provided you give appropriate credit to the original author(s) and the source, provide a link to the Creative Commons license, and indicate if changes were made. The Creative Commons Public Domain Dedication waiver (http://creativecommons.org/publicdomain/zero/1.0/) applies to the data made available in this article, unless otherwise stated. 


\section{Introduction}

In 2018, 1,316,350 children attended Early Childhood Education and Care (ECEC) services in Australia [1]. This figure includes children who attended Long Day Care (LDC), Family Day Care and Outside of School Hours Care. In 2018, 751,450 children were in LDC and, on average, spent $25.7 \mathrm{~h}$ per week in care [1]. LDC has the highest proportion of children (57.2\%) compared to other service types [1]. Typically, these are services that provide children with education and care for more than $8 \mathrm{~h}$ a day, 5 days a week and include meals (specifically morning tea (MT), lunch (L) and afternoon tea (AT)) prepared on site. Thirty seven percent of all children aged 2-3 years attended formal care which was most likely to be LDC [2]. Nationally, demand for formal care exceeds supply; currently, the deficit is approximately 9\% [2]. Hence, there is a pressing need to grow this sector which also makes good economic sense, amounting to a $3.9 \%$ increase valued at $\$ 12$ billion per year [3].

The ECEC sector provides education and support for children at a rapid stage of growth and development; which coincides with the formation of dietary habits [46]. Establishing healthy dietary habits at an early age that support healthy food preferences, has been shown to continue into adulthood and reduce the risk of obesity and non-communicable disease [7-10]. Given this critical time point and the number of children being provided food in LDC, there is an urgent need to ensure that food provision at a service level complies with current dietary guidelines and is accurately assessed.

There have been various intervention strategies implemented in past decades to improve food provision in LDC which have involved face to face training and award schemes [7, 8, 11-14]. The Australian Dietary Guidelines (ADG) have based recommended intakes on core food groups primarily because people eat food not nutrients. These guidelines also provide recommendations for children under 4 years [15]. More recently, interventions have been based on encouraging compliance with the current ADG (2013) [16, 17], which recommend compliance in provision of food group serves for children from 6 months of age. To monitor compliance with dietary recommendations, food group provision needs to be measured at the service level to ensure that, on average, children are being provided with the correct number of servings of food groups and that there is an opportunity to consume the recommended serves of food groups appropriate for the child's age and gender.

Research suggests that children attending LDC should consume at least $50 \%$ of their daily food intake during morning tea (MT), lunch (L) and afternoon tea (AT) [18-21] and reports have indicated that children consume between 40 and $67 \%$ of their food intake whilst attending LDC services $[11,22,23]$. This makes LDC settings ideal venues to ensure dietary guidelines are being met and to positively influence the nutritional status of children [24-27].

There is, however, limited evidence of whether food provision in LDC meets current dietary guidelines in Australia. Assumptions of inadequate food provision are typically based on limited reviews of menus [28, 29], food budgets [28], observation methodology [30, 31] rather than "actual" provision, determined from measuring ingredient weights across a range of meals and days at a service $[28,32,33]$. The underpinning premise of measuring food provision is based on the understanding that if core food groups are underprovided (compared to dietary guideline food group serve recommendations), then food groups, most likely, will be "under consumed". Hence it is unlikely that the recommended food group serves would be met by an individual child, jeopardising health and developmental benefits from optimal nutrition.

Historically, Australian government and nongovernment organisations have prioritised nutrition in LDC, yet the limited and varied evidence available from the last two decades suggests little improvement in compliance with dietary guidelines or serve recommendations [22, 34, 35]. In addition, the change of serve size and recommended number of serves between 2003 and 2013 [35, 36], the lack of recommended procedures for monitoring food provision, and limited research, makes comparison of research from different studies over time difficult and less likely to provide an accurate representation of trends and the current status [37].

It is generally recognised that the "gold standard" for determining individual food intake is a weighed food record [37-39]. At a service level, there is currently limited evidence of accurate estimates of food provision nor is there a recommended "gold standard" [8, 40]. This protocol paper intends to bridge this gap by contributing knowledge towards the development of a gold standard measure. Specifically, it provides a data collection method used to measure food group provision and food waste in LDC services based on weighed food record at a service level. This will be useful for researchers, industry and resource development by providing evidence to help understand food provision at a service level, monitor change across time and allow for comparative studies based on a common methodology.

\section{Evidence of variation of data collection methods}

Research has determined that food provided in the ECEC sector from Australia [34], USA [41-43] and the Netherlands $[44,45]$ does not generally meet respective nutritional requirements for children based on dietary recommendations for the individual. Moreover, previous researchers have used different methodologies to determine the adequacy of food provision. These include: 
individual food records to determine foods and/or nutrient provision or intake [33, 45, 46]; food provision assessment by menu review [28, 29, 47]; food intake by survey or self-reporting [26, 48, 49]; and plate wastage [17].

In addition, the methods to determine food provision vary between studies [13, 50-52]. From this perspective, the issues are two-fold: comparison between research findings cannot be truly accurate if different data collection methods were used; and that these methodologies, such as, menu reviews, observations, surveys, may not provide a true reflection of the actual food provided, which limits the ability of these studies to accurately assess food provision [30, 31]. Further evidence of variation in data collection methods and limitations are summarised below.

\section{Observation method}

Erinosho et al. [53] used the Healthy Eating Index-2005 (HEI-2005) to evaluate foods offered in 20 LDC in North Carolina, United States. Food serves were recorded by an observation method to determine individual dietary intake. Direct observation of dietary intake is often a means of validating a dietary assessment method. Trained observers note down, but not limited to, eating behaviours, food ingredients and portions during a nominated period. This method is not considered a suitable level to determining a habitual dietary pattern at an individual or group level [54]. The total mean score of food and beverages provided to children in day care services was significantly lower than the optimal recommended score $(59.12$ vs optimal score $100 ; P<0.01)$. Despite their belief that observation was a suitable method to determine individual food intake, the 8 year delay in data analysis from 2005 to 2013 is a major limitation as child care practices may have altered. In a further study, Padget and Briley [55] used observation combined with weight estimation with measuring cups as a method to determine individual food consumption. The researchers also considered this a suitable method despite commentary regarding increasing the days of observation and the numbers of children would improve the quality of their findings.

Benjamin et al. [29] compared menus in child care with state dietary regulations and found regulations regarding menus varied from state to state. While this type of research is important for determining whether services are following policy guidelines, the issue of jurisdictional differences may confound results.

A study by Henderson et al. [56] sought to develop a validated tool that could measure nutriton in LDC settings, which included food observation and a scoring system for unhealthy and healthy foods. This study also utilised a menu rating tool and service director interviews. The authors noted the study limitiations as poor generalisabilty, only a single day of observation and potential social desirablity bias.

\section{Menu review}

In 2018, research was published that examined sodium intake from snacks provided in LDC, where meals were photographed pre and post meal times and consumption was estimated. Menus (recipes) were collected for 5 days and evaluated using the Healthy Eating Advisory Service Guidelines (Victoria, Australia) [57]. This research highlighted that recipes from proposed menus differed from actual in service food provision [58], thus, menus can be a poor reflection of actual food provision.

In 2017, a study conducted in New Zealand utilised an online survey tool, email or facsimile to share menus. The results found that most food groups did not meet nutrition guidelines. The study limitations included utilisation of self-reported menus and recipes and found that these did not necessarily reflect food that was provided or consumed [28]. This has been further supported by other research emphasising menu reviews may not reflect actual food provision [30,31].

A more recent Australian study was designed to assess foods provided by LDC and compared these to the ADG recommendations utilising a review of a two-week menu plan to calculate the number of serves of vegetables, fruit, grains, meat and dairy [15]. Descriptive statistics showed no centre met the recommendations for vegetables, $96 \%$ for fruit, $87 \%$ for grains, $59 \%$ for meat and $89 \%$ for dairy [49]. This same study reported that meals differed from what food was actually provided and the focus for this research was on an individual child's consumption, rather that food provision at a service level.

\section{Self-reporting}

A study by Benjamin-Neelon et al. [48] assessed 13 guidelines that related to restricting or encouraging foods and compared these nutrition practices with voluntary national guidelines for the Australian equivalent of LDC. However, they did not measure food groups or nutrient quantities but whether a food, e.g. a vegetable, was served. This study highlighted significant jurisdictional differences of recommendations in the United States. A limitation of the study, was the reliance on self-reporting food provision by LDC rather than an objective measure of dietary assessment which likely resulted in the overestimation of foods due to a social desirability bias. Further to this a protocol paper by Yoong et al. [59], planned to pilot the effectiveness of an online web based tool, which also relies on selfreporting. 
Food frequency questionnaires (FFQ)/food recalls (FR) A systematic review [58] was conducted to determine the validity of dietary assessment methods used to determine energy intake in overweight or obese children. This study found that Food Recalls (FR) were repeatedly inaccurate across all age groups and reporters. In addition, a systematic review of the accuracy and reliability of methods used for assessing an individual child's diet in the school context, found the outcomes were also variable; which supports other research findings to date [60].

Lovell et al. [61] conducted a systematic review on validating Food Frequency Questionnaires (FFQ). This study reviewed 17 articles and found further validation of appropriate population specific tools addressing portion size estimation for young children was required. This review found that FFQ overestimated intakes and individual consumption methods recommended a 3-5 day snapshot of food intake to increase reliability [62]. To the authors knowledge, there has been no systematic review conducted to assess FR/FFQ accuracy and reliability in LDC.

\section{Portion size}

Traditional strategies of measuring food intake can have a high participant burden. To minimise this, the use of portion size assessment tools to estimate individual food consumption has been suggested [37]. This method relies on rigorous training of researchers or service staff and captures an individual's food provision or consumption. Foster et al. [37], also suggested the nature of the research setting may increase the awareness of the foods being consumed, thus, not accurately representing food provision.

\section{Plate waste}

An older study by Soanes et al. [23], weighed food and deducted the waste to determine individual consumption over three consecutive days and compared this to recommended dietary intakes (RDI) for a range of macro and micro nutrients. This study established that none of the children attending LDC were consuming $50 \%$ of their respective RDI whilst attending LDC.

Recently, Seward et al. [63] conducted research on a multi strategy intervention to improve food provision at a service level. Multiple intervention strategies were used to ascertain measures including, aggregated plate waste measures to assess consumption and utilised menus, self-reported by LDC, to determine provision. This study recommended that multiple observation periods be incorporated to better reflect usual child food intake.

There are limitations with these methods. Dietary recording conducted at an individual child level [21] or use of FFQ, often overestimate provision [64] due to social desirability bias $[65,66]$. Thompson and Subar [38], confirmed that self-reporting, including FFQ and FR, were problematic as a result of bias for both sample and number of days recorded. The same authors suggest technology [67] should be incorporated to more accurately capture food intake. Further to this, research participants have utilised mobile phones or tablets to capture food images consumed at meal times [68], which can improve accuracy of data collection methods.

In 2014, a pilot study [34], believed to be the first Australian study to examine food provision by food group in LDC services, compared foods provided by a service with $50 \%$ of serve recommendations based on the ADG [15]. The authors analysed food and beverages provided to children 2-4 years old from eight LDC services. They weighed all foods and converted them to food groups by a predetermined formula, which converted ingredients into grams of respective food groups. Their analysis showed a significant difference in the number of meat or meat alternatives serves compared to $50 \%$ of the ADG $(0.33 \pm 0.20$ serves compared to 0.50 serves; $P=0.05)$. There was no significant difference in the other food groups; however, dairy and fruit exceeded the recommended $50 \%$ of serves [34]. This pilot study was conducted to test the suitability of a weighed food data collection method at the service level and found weighing individual ingredient to be an accurate method of data collection.

In summary research highlights international and national inconsistencies when measuring the provision of foods in LDC. Furthermore, at a service level, there is limited research on measurement of food provision. An agreed method of measurement for food provision in LDC services would improve the reporting of food compliance outcomes of services and provide a platform to design resources that can form the basis of consistent recommendations across jurisdictions. A pilot study using an approach considered gold standard for measuring dietary intake in individuals, was deemed a suitable method to accurately determine food provision at a service level [34]. Therefore, this paper builds on that work by providing the methodology for a weighed food data collection method to measure food provision and waste at a service level.

\section{Methods \\ Aim}

To propose a suitable method of data collection to accurately report on food provision status of LDC services.

\section{Study design}

A cross-sectional audit in LDC services, in metropolitan Perth, Australia, was conducted by weighing the raw ingredients provided at each meal time; morning tea, 
lunch and afternoon tea (MT, L, AT), to determine food group provision. This study was approved by the Human Research Ethics Committee (HREC), Edith Cowan University (\# 18486).

The data was collected over a three-year period; 2015, 10 services; 2016, 9 services and 2017, 11 services, providing a total sample of 30 services. A required minimum sample size of 27 services was determined using the Wilcoxon signed-rank test at a $5 \%$ level of significance and $80 \%$ power, with a medium (Cohen's $d=0.5$ ) effect size. This sample provided sufficient numbers to detect an improvement in the proportion of services (from 40 to $80 \%$ ) meeting $50 \%$ food group compliance based on ADG recommendations [15].

\section{Participants and recruitment}

The sample population included LDC services which operated for more than $8 \mathrm{~h}$ per day, 5 days a week and prepared food on site. The services were included if they were located in the Perth, Western Australia metropolitan area (postcodes between 6000 and 6199) and were not previously involved in the pilot study [34]. Services were randomly selected via a publicly available register accessed from Australian Children's Education and Care Quality Authority (ACECQA) [69], where every 10th centre was telephoned and asked to particpate in the study (maximum follow up attempts 3 ). If the services met the inclusion criteria, and they agreed to participate, an information letter was sent and followed with a telephone call 2 days later to schedule suitable days for data collection.

\section{Exclusion criteria}

LDC services were excluded from the study if they had participated in the pilot study or in previous years, they declined, or were outside of metropolitan Perth.

\section{Process of data collection and data entry}

Figure 1, represents the preparation and process for data collection and data entry.

\section{Research assistant training (Fig. 1, item 1)}

Prior to the data collection, research assistants, which included undergraduate Nutrition and postgraduate $\mathrm{Nu}$ trition and Dietetics students, underwent $4 \mathrm{~h}$ of supervised training [60] to ensure standardised data collection protocol and to become familiar with food preparation in the LDC setting, food measurement using calibrated scales and to familiarise researchers with data recording methods. Training included demonstration and practical application of the major daily tasks outlined in Table 1 below, including training of research assistants to use Foodworks 9, a nutrient analysis Australian software program [70].

In addition a comprehensive online interactive food safety training package from Environmental Health Australia "I'M ALERT" was completed [71], which included 23 sections on basic food safety principles.

Contact service and request menu and recipes (Fig. 1, item 2) Research assistants contacted the LDC 2 weeks prior to scheduled site visit to request the menu and recipes that would be prepared on two consecutive data collection

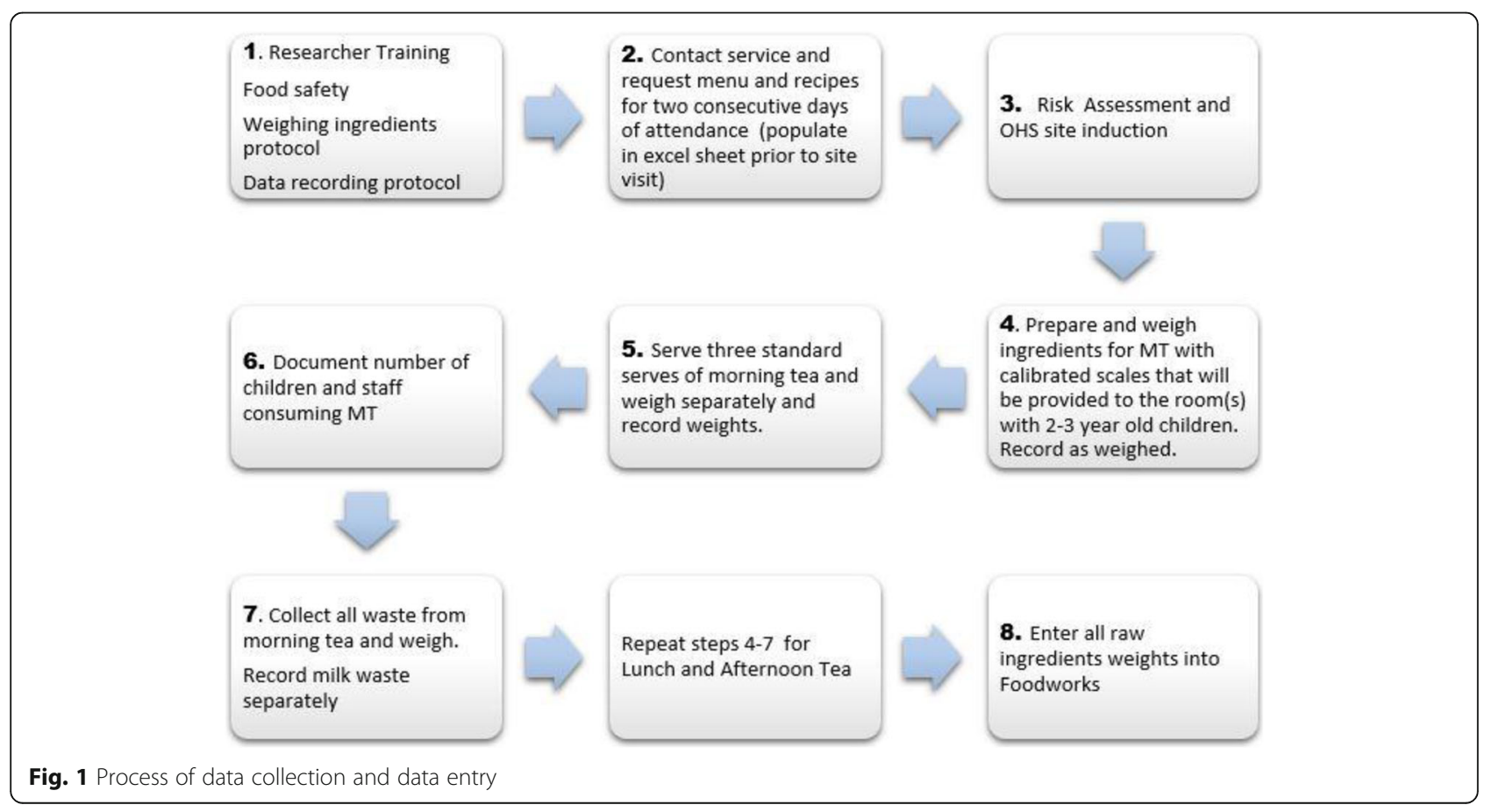


Table 1 : Summary of major daily tasks, on site, of research assistants

\begin{tabular}{|c|c|}
\hline Time & Task \\
\hline Pre - day 1 or prior to site visit & $\begin{array}{l}\text { 1. Undertake orientation at facility including policy/procedures and OHS requirements, } \\
\text { certificate of currency and risk assessment. } \\
\text { 2. Meet with manager/kitchen staff. }\end{array}$ \\
\hline $\begin{array}{l}\text { Day } 1 \text { and } 2 \text { (consecutive days): Data } \\
\text { Collection }\end{array}$ & $\begin{array}{l}\text { 1. Arrive early, prepare and weigh individual morning tea ingredients and record into a pre populated MS } \\
\text { Excel spreadsheet. } \\
\text { 2. Record children and staff (only if consuming meals) meal attendee numbers } \\
\text { 3. Weigh three standard serve sizes - to determine the average meal weight } \\
\text { 4. Collect waste from morning tea, weigh and record into a pre populated MS Excel spreadsheet. } \\
\text { 5. Prepare and weigh individual lunchtime ingredients and record into a pre populated MS Excel } \\
\text { spreadsheet. } \\
\text { 6. Record children and staff (only if consuming meals) meal attendee numbers } \\
\text { 7. Weigh three standard serve sizes to determine the average meal weight } \\
\text { 8. Collect waste from lunchtime, weigh and record into a pre populated MS Excel spreadsheet. } \\
\text { 9. Prepare and weigh individual afternoon tea ingredients and record into a pre populated MS Excel } \\
\text { spreadsheet. } \\
\text { 10. Record children and staff (only if consuming meals) meal attendee numbers } \\
\text { 11. Weigh } 3 \text { standard serve sizes to determine the average meal weight } \\
\text { 12. Collect waste from afternoon tea, weigh and record into a pre populated MS Excel spreadsheet. } \\
\text { 13. Repeat this procedure for Day } 2 \text {. }\end{array}$ \\
\hline Day 3 & Enter food ingredients and weights into Foodworks \\
\hline
\end{tabular}

days. These recipes were pre-populated into an Excel spreadsheet including the number of anticipated serves. On the day of data collection, the actual weight of the recipe ingredients and number of attendees were documented in a separate column for comparison at a later date.

\section{Risk assessment and induction (Fig. 1, item 3)}

Prior to data collection days, Occupational Health and Safety (OHS) induction was completed. This process included familiarisation of the centre, emergency procedures, and completion of risk management forms and exchange of certificate of currency for insurance.

\section{Preparation and weighing raw ingredients (Fig. 1, item 4)}

Day 1 and 2: The raw ingredients used to prepare MT, L and AT meals were weighed with calibrated scales (A\&D Australasia, SJ-5001HS) and recorded over two consecutive days. As most services have multiple rooms, the weight of the ingredients had to reflect proportionately what was offered to each room. The weight, in grams, of all ingredients provided, for each room, were entered into the pre-populated Microsoft Excel sheet in the "actual" food weight column. It was deemed by the chief investigators that data gathering for all age groups would allow for more accurate reflection of provision for children 2-3 years at a later date if required.

Day 3: Each raw ingredient weight was added for each recipe, where there were multiple recipes for a meal occasion, these were added separately as was milk (full cream unless otherwise specified), into Foodworks [70]. The number of children and staff that were consuming food at each meal time was also added for each recipe to Foodworks. The recipes were used to populate a daily meal plan for a reference child: $2-3$ year old boy, including; MT, L and AT. A 2-3 year old boy was chosen as a reference child in Foodworks as the highest proportion (approx. 45\%) of children attending LDC are aged 2-3 years [72] and for children aged 2-3 years there is no gender difference between food group serve size recommendations [36].

Where product items were unknown or not recognised by Foodworks a standard equivalent item was chosen, to reduce discrepancies in data collation.

Foodworks [70] data was exported into Microsoft Access [73] and then into IBM SPSS Statistics [74]. Food group and nutrient line data, by child, by day, by service, were available for analysis.

Included in the analysis was discretionary food, which is described as foods and drinks that do not fit into the Five Food Groups because they are not considered a part of a healthy diet as they are too high in saturated fat and/or added sugars, added salt or alcohol and low in fibre [75]. Where discretionary foods were identified the individual ingredient weights were converted to a serve based on the Australian Dietary Guideline recommendations (one serve of discretionary food $=600 \mathrm{~kJ}$ ) [75]. The discretionary food serves per meal were added separately to the final SPSS data file for each meal time as this was not captured by Foodworks. Further to, the fat allowance was based on the ADG [15]. In addition, Nutrient Reference Values [76], provided guidelines for fat as a percentage of energy intake and average intake allowances for a variety of fatty acids for comparative analysis.

A one-sample t-test or Wilcoxon signed-rank test was used to compare food provision to the 50\% recommended serves of each food category, discretionary serve 
Table 2 50\% of recommended serves of food groups, fat allowance and discretionary foods, for 2-3 year olds based on ADG [15]

\begin{tabular}{lll}
\hline Food category & Recommended serves for 2-3 year olds [15] & $50 \%$ Recommended serves \\
\hline Vegetables & 2.5 & 1.25 \\
Fruit & 1 & 0.50 \\
Grains/cereals & 4 & 2.00 \\
Milk/milk alternatives & 1.5 & 0.75 \\
Lean meat/meat alternatives & 1 & 0.50 \\
Fat allowance & $35-40 \%$ of TEl $^{\text {a }}$ & $35-40 \%$ of TEl $^{\text {a }}$ \\
Discretionary foods & $0.00^{\mathrm{b}^{\mathrm{b}}}$ & $0.00^{\mathrm{b}}$ \\
\hline
\end{tabular}

${ }^{a}$ Fat allowance based on 35-40\% of Total Energy Intake (TEI) [35]

${ }^{b}$ Eat For Health recommends that discretionary foods should not be offered in ECEC services [75]

and fat allowance for children aged 2-3 years (Table 2) [36]. $P$ value $\leq 0.05$ was assumed statistically significant.

Descriptive statistics were be utilised to describe the data between the two consecutive days, the average of data collection and between the services for each food category. Shapiro-Wilk test will be used to verify normality.

\section{Weigh three standard serves of meal time and record weights (Fig. 1, item 5)}

Kitchen staff/educators were asked to serve up three standard meals that would represent a typical serving offered to the room, three standard plates were tared. Meal weights were documented then averaged. This average weight was recorded as a standard serve for the specified meal time. The standard serve was used to calculate proportion of ingredients of cooked weight at a later date if required, particularly where leftovers were recorded. Leftovers were considered as any food that was held back to be served at a later stage that would affect the total weight of food that had been weighed for a specific meal time. Leftovers that are part of the original total weight of ingredients are weighed and this weight is documented minus storage containers, to give a total weight of ingredients that can be deducted from a specific meal time.

Research assistants are required to document the volume of milk that was allocated to the room and then document the volume of milk that is returned and will be offered later at a different meal time. The total milk minus the volume of milk that will be used at a later stage will equal the figure entered into Foodworks as provision, milk waste should be added as per Fig. 1, item 7 .

\section{Document number of children and staff consuming MT}

\section{(Fig. 1, item 6)}

The number of children and staff sharing the meal in the room for each specific meal time (MT, L and AT) are counted, documented and utilised for food group provision calculations for each meal time.

\section{Measuring waste (Fig. 1, item 7)}

Aggregated waste was measured for each meal time excluding leftovers, following the steps below;

1. Place a plastic bag inside a bucket and record the weight.

2. Scrape all food from plates from the relevant room, into the bucket and record weight, check floors and tables for any additional waste for inclusion.

3. Weight of leftovers (measure milk separately) are recorded separately.

4. Repeat for each mealtime.

5. At the end of the day, reweigh the bucket containing the scraps and record second weight, average and enter into the excel sheet.

6. Milk waste should be collected in a jug (tared) to measure total waste for each meal time and recorded separately to food waste weight.

\section{Reliability}

Reliability of the data collection process relates to the consistency in training of all research assistants prior to data collection ensuring adherence to the protocol thus, quality of data. As this data collection method measured actual weight of food ingredients there was limited opportunity for variability of actual weights and social desirability bias. A second set of calibrated scales were available if required. Validity could have been increased if raw ingredients were re-weighed three times and an average taken. This was not practical due to time constraints around meal preparation in LDC service kitchens. Reliability of analysis was increased by entering all data into same version of Foodworks.

\section{Strengths and limitations}

- Training of researchers reduces the variability of data collection and is a critical part of the process.

- A weighed food ingredient measure provides an accurate representation of food provided at a service level. 
- This method is less onerous on individuals and services, which increases the level of willingness to participate in the study.

- Data is collected across two consecutive days, increasing the number of days would increase the transferability of the outcomes.

- Services were also aware they were participating in research and may potentially supplement/alter ingredients, which could skew findings due to social desirability bias.

\section{Discussion}

The method of data collection is critical to accurately measure food provision in LDC. The variation in research methods limits comparability of food provision over time, yet historically these research projects are used to determine and compare compliance of food provision in LDC. Food weight records are deemed gold standard when determining individual food consumption $[32,39]$, yet there is a paucity of evidence for accurately measuring food provision at a service level in LDC. Dietary assessment pertaining to weighed food records were adapted for a service setting and were more accurately able to reflect food group provision. We recommend that the measurement of LDC food provision data, be captured using this method in order to more effectively determine change, compare studies over time and report on the current status of food provision at a service level which could be extended to a national bi-annual audit in LDC. An audit would enable food provision surveillance at a service, state and national level and could guide intervention strategies and resource development in a more targeted way. The audit could be coordinated collaboratively between an accrediting body, such as ACECQA and the tertiary sector, with specific oversight from early years nutrition specialists such as members of the National Nutrition Network-Early Childhood Education and Care (NNN-ECEC), whose mission is to promote best practice provision of food within ECEC services to facilitate positive short and long term nutrition, health and development outcomes for children who attend care.

\section{Abbreviations}

ADG: Australian dietary guidelines; AT: Afternoon tea; ECEC: Early childhood education and care; FFQ: Food frequency questionnaire; FR: Food recall; L: Lunch; LDC: Long daycare; MT: Morning tea

\section{Acknowledgements}

Thank you to research assistants, Andrea Gracie, Melissa Dunham, Jacqueline Van Zyl, Clare Richards, Julia Main, Michaela Johnston and Krystal Kotkis. Thank you to the 30 services who participated in the research.

\section{Authors' contributions}

Sambell was the lead researcher, Devine, Wallace and Costello provided guidance ongoing support and were major contributors to manuscript. Lo was a statistician and provided ongoing research and analysis guidance and manuscript contribution on same. All authors contributed to the critical review of the manuscript. All authors read and approved the final manuscript.

Funding

No funding was gained to support this research.

Availability of data and materials

This paper is a study protocol hence no linked data.

Ethics approval and consent to participate

18486 Edith Cowan University, Western Australia.

Consent for publication

Not applicable.

\section{Competing interests}

The authors declare that they have no competing interests.

Received: 18 December 2018 Accepted: 25 June 2019

Published online: 16 July 2019

\section{References}

1. Department of Education Employment and Workplace Relations. Child Care in Australia Canberra: Department of Educaiton and Training; 2018. Available from: https://www.education.gov.au/child-care-australia. [cited 201916 May]

2. Australian Bureau of Statistics. Care usually attended Australia: ABS; 2018. Available from: http://www.abs.gov.au/ausstats/abs@.nsf/mf/4402.0. [cited 2018 19th July]

3. Ibis world. Child care services - Australia market research report 2018. Available from: https://www.ibisworld.com.au/industry-trends/marketresearch-reports/health-care-social-assistance/child-care-services.html. [cited 2018 July].

4. Pietrobelli A, Agosti M. The MeNu group. Nutrition in the first 1000 days: ten practices to minimize obesity emerging from published science. Int J Environ Res Public Health. 2017;14(12):1491.

5. Australian Institute of Health and Welfare. Australia's welfare 2015. Australia's welfare series no 12 Cat no AUS 189. Canberra: AlHW; 2015.

6. Moore TG, Arefadib, N., Deery, A., Keyes, M. \& West, S. The first thousand days: an evidence paper - summary. 2017. Available from: http://apo.org.au/ system/files/108431/apo-nid108431-436656.pdf.

7. Golley R, Bell L, Matwiejczyk L, Hartley J. South Australian long day care Centres engaged with a nutrition incentive award scheme show consistency with mealtime practice guidelines. Nutr Diet. 2012;69:130-6.

8. Matwiejczyk L, Colmer K, McWhinnie J. An evaluation of a nutrition intervention at childcare centres in South Australia [online]. Health Promot Austr. 2007;18(2):159-62.

9. Larson N, Ward D, Benjamin Neelon S, Story M. What role can child-care settings play in obesity prevention? A review of the evidence and call for research efforts. J Am Diet Assoc. 2011;111(9):1343-62.

10. Buscemi J, Kanwischer K, Becker AB, Ward DS, Fitzgibbon ML, On behalf of the Society of Behavioral Medicine Health Policy C. Society of Behavioral Medicine position statement: early care and education (ECE) policies can impact obesity prevention among preschool-aged children. Transl Behav Med. 2015:5(1):122-5.

11. Finch M, Seward K, Wedesweiler T, Stacey F, Grady A, Jones J, et al. Challenges of increasing childcare center compliance with nutrition guidelines: a randomized controlled trial of an intervention providing training, written menu feedback, and printed resources. Am J Health Promot. 2018;33(3):399-411

12. Jones J, Wyse R, Finch M, Lecathelinais C, Wiggers J, Marshall J, et al. Effectiveness of an intervention to facilitate the implementation of healthy eating and physical activity policies and practices in childcare services: a randomised controlled trial. Implement Sci. 2015;10:147.

13. Yoong SL, Jones J, Marshall J, Wiggers J, Seward K, Finch M, et al. A theorybased evaluation of a dissemination intervention to improve childcare cooks' intentions to implement nutritional guidelines on their menus. Implementat Sci. 2016;11(1):105.

14. Matwiejczyk L, Mehta K, Scott J, Tonkin E, Coveney J. Characteristics of effective interventions promoting healthy eating for pre-schoolers in childcare settings: an umbrella review. Nutrients. 2018;10(3):293. 
15. National Health and Medical Research Council. In: Department of Health and Ageing, editor. Australian Dietary Guidelines (ADG). Canberra: National Health and Medical Research Council; 2013.

16. Bell CA, Davies L, Finch M, Wolfenden L, Francis L, Sutherland R, et al. An implementation intervention to encourage healthy eating in Centre-based child-care services: impact of the good for kids good for life programme. Public Health Nutr. 2015;18(09):1610-9.

17. Bell LK, Hendrie GA, Hartley J, Golley RK. Impact of a nutrition award scheme on the food and nutrient intakes of 2- to 4-year-olds attending long day care. Public Health Nutr. 2015;18(14):2634-42.

18. Baade PD, Radcliffe BC, Cameron CV. Nutrient intakes of young children: implications for long-day child-care nutrition recommendations. Nutr Diet. 2002;59(3):187.

19. NSW Ministry of Health. In: Ministry of Health, editor. Caring for Children Birth to 5 years (Food, Nutrition and learning experiences). NSW: Ministry of Health; 2014.

20. Benjamin Neelon S, Briley M. Position of the American dietetic association: benchmarks for nutrition in child care. J Am Diet Assoc. 2011;111(4):607-15.

21. Radcliffe BC, Cameron CV, Baade PD. Nutrient intakes of young children: implications for long-day child-care nutrition recommendations. Nutr Diet. 2002:59(3):187-90 4.

22. Pollard C, Lewis J, Miller M. Food service in long day care centres - an opportunity for public health intervention. Aust N Z J Public Health. 1999; 21:638-42.

23. Soanes R, Miller M, Begley A. Nutrient intakes of two- and three-year-old children: a comparison between those attending and not attending long day care centres. Aust J Nutr Diet. 2001;58(2):114-20.

24. Lombardi K. Design, implementation and evaluation of the impact of a body image professional development program for early childhood educators. Perth: Edith Cowan University, Edith Cowan University, Research Online; 2018.

25. TO E, Hales DP, McWilliams CP, Emunah J, Ward DS. Nutrition policies at child-care centers and impact on role modeling of healthy eating behaviors of caregivers. J Acad Nutr Diet. 2012;112(1):119-24.

26. Ward D, Mazzucca S, McWilliams C, Hales D. Use of the environment and policy evaluation and observation as a self-report instrument (EPAO-SR) to measure nutrition and physical activity environments in child care settings: validity and realiability evidence. Int J Behav Nutr Phys Act. 2015;12:124.

27. Wallace R. Supporting nutrition for Australian childcare (SNAC): the development, implementation and evaluation of an online nutrition education intervention. Perth: Edith Cowan University, Edith Cowan University, Research Online; 2016.

28. Gerritsen S, Dean B, Morton SMB, Wall CR. Do childcare menus meet nutrition guidelines? Quantity, variety and quality of food provided in New Zealand early childhood education services. Aust N Z J Public Health. 2017;41(4):345-51.

29. Benjamin SE, Copeland KA, Cradock A, Neelon B, Walker E, "Slining MM, et al. Menus in child care: a comparison of state regulations with national standards. J Am Diet Assoc 2009;109(1):109-115.

30. Fleishhacker S, Cason KL, Achterberg C. "You had peas today?": a pilot study comparing a head start child-care center's menu with the actual food served. J Am Diet Assoc. 2006;106:277-80.

31. Benjamin-Neelon SE, Copeland K, Ball SC, Bradley L, Ward DS. Comparison of menus to actual foods and beverages served in North Carolina child-care centers. J Am Diet Assoc. 2010;110(12):1890-5.

32. Tufts University Friedman School of Nutrition Science and Policy Web and Communications. Data 4Diets :Weighed Food Record (WFR)2018. Available from: https://inddex.nutrition.tufts.edu/data4diets/data-source/weighedfood-record-wfr. Accessed Nov 2018.

33. Ishii $Y$, Ishihara J, Takachi R, Shinozawa Y, Imaeda N, Goto C, et al. Comparison of weighed food record procedures for the reference methods in two validation studies of food frequency questionnaires. J Epidemiol. 2017;27(7):331-7.

34. Sambell R, Devine A, Lo J. Does the food group provision in early years' education and care settings in metroploitan Perth, Western Australia, meet national dietary requirements; and how can home economics support this? J HEIA. 2014;21(2):20-7.

35. National Health and Medical Research Council (NHMRC). NHMRC, editor. Dietary guidelines for children and adolescents in Australia: incorporating the infant feeding guidelines for health workers. Canberra: Commonwealth of Australia. p. 2003.
36. National Health and Medical Research Council (NHMRC). Australian dietary guidelines (2013). In: NHMRC, editor. . Canberra: Commonwealth of Australia; 2013.

37. Foster E, Hawkins A, Barton KL, Stamp E, Matthews JNS, Adamson AJ. Development of food photographs for use with children aged 18 months to 16 years: comparison against weighed food diaries - the young Person's food atlas (UK). PLoS One. 2017;12(2):e0169084.

38. Thompson FE, Subar AF. Chapter 1 - dietary assessment methodology. In: Coulston AM, Boushey CJ, Ferruzzi MG, Delahanty LM, editors. Nutrition in the prevention and treatment of disease. 4th ed. United States: Academic Press; 2017. p. 5-48.

39. Carlsen MH, Lillegaard ITL, Karlsen A, Blomhoff R, Drevon CA, Andersen LF. Evaluation of energy and dietary intake estimates from a food frequency questionnaire using independent energy expenditure measurement and weighed food records. Nutr J. 2010;9:37.

40. Moore H, Nelson P, Marshall J, Cooper M, Zambas H, Brewster K, et al. Laying foundations for health: food provision for under $5 \mathrm{~s}$ in day care. Appetite. 2005:44(2):207-13.

41. Zuercher J, Grace E, Kranz S. Comparing diet quality in child care center menus after revision. Child Obes. 2011;7(5):392-9.

42. Ritchie LD, Boyle M, Chandran K, Spector P, Whaley SE, James P, et al. Participation in the child and adult care food program is associated with more nutritious foods and beverages in child care. Child Obes. 2012:8(3):224-9.

43. Maalouf J, Evers SC, Griffin M, Lyn R. Assessment of mealtime environments and nutrition practices in child care centers in Georgia. Child Obes. 2013; 9(5):437-45.

44. Gubbels JS, Kremers SPJ, Stafleu A, Dagnelie PC, De Vries NK, Thijs C. Childcare environment and dietary intake of 2- and 3-year-old children. J Hum Nutr Diet. 2009;23(1):97-101.

45. Goldbohm RA, Rubingh CM, Lanting $\mathrm{Cl}$, Joosten KFM. Food consumption and nutrient intake by children aged 10 to 48 months attending day care in the Netherlands. Nutrients. 2016;8(7):428.

46. Chen C, Denney L, Zheng Y, Vinyes-Pares G, Reidy K, Wang H, et al. Nutrient intakes of infants and toddlers from maternal and child care centres in urban areas of China, based on one 24-hour dietary recall. BMC Nutrition. 2015;1(1):23.

47. Benjamin-Neelon SE, Reyes-Morales H, Haines J, Gillman MW, Taveras EM. Nutritional quality of foods and beverages on child-care Centre menus in Mexico. Public Health Nutr. 2013;16(11):2014-22.

48. Benjamin-Neelon SE, Burgoine $T$, Hesketh KR, Monsivais P. Nutrition practices of nurseries in England. Comparison with national guidelines. Appetite. 2015:85:22-9.

49. Yoong L, Skelton E, Jones J, Wolfenden L. Do childcare services provide foods in line with the 2013 Australian dietary guidelines? A cross-sectional study. Aust N Z J Public Health. 2014;38(6):595-6.

50. Oakley CB, Bomba AK, Knight KB, Byrd SH. Evaluation of menus planned in Mississippi child-care centers participating in the child and adult care food program. J Am Diet Assoc. 1995;95(7):765-8

51. Krukowski RA, Eddings K, Smith West D. The Children's menu assessment: development, evaluation, and relevance of a tool for evaluating Children's menus. J Am Diet Assoc. 2011:111(6):884-8.

52. Coates J, Colaiezzi B, Fiedler J, Wirth J, Lividini Ka, Rogers B. Applying dietary assessment methods for food fortification and other nutrition programs; 2012. Available from: http://www.harvestplus.org/sites/default/files/ Dietary\%20Assessment\%20Methods_Sept\%202012.pdf.

53. TO E, Ball SC, Hanson PP, Vaughn AE, Ward DS. Assessing foods offered to children at child-care centers using the healthy eating Index-2005. J Acad Nutr Diet. 2013;113:1084-9.

54. University of Cambridge. DAPA measurement toolkit: direct observation. Available from: http://dapa-toolkit.mrc.ac.uk/diet/objective-methods/directobservation. [cited 2018 9th November]

55. Padget A, Briley ME. Dietary intakes at child-care centers in Central Texas fail to meet food guide pyramid recommendations. J Am Diet Assoc. 2005;105(5):790-3.

56. Henderson KE, Grode GM, Middleton AE, Kenney EL, Falbe J, Schwartz MB. Validity of a measure to assess the child-care nutrition and physical activity environment. J Am Diet Assoc. 2011;111(9):1306-13.

57. Healthy Eating Advisory Service. Early childhood services 2018. Available from: https://heas.health.vic.gov.au/. [cited 2018 august]

58. O'Halloran SA, Lacy KE, Grimes CA, Campbell KJ, Nowson CA. Sodium content of lunches and snacks provided in Australian long day care Centres: a cross-sectional study. Nutrients. 2018;10(3):284. 
59. Yoong SL, Grady A, Wiggers J, Flood V, Rissel C, Finch M, et al. A randomised controlled trial of an online menu planning intervention to improve childcare service adherence to dietary guidelines: a study protocol. BMJ Open. 2017;7(9):e017498.

60. Tugault-Lafleur CN, Black JL, Barr SI. A systematic review of methods to assess Children's diets in the school context. Adv Nutr. 2017;8(1):63-79.

61. Lovell A, Bulloch R, Wall CR, Grant CC. Quality of food-frequency questionnaire validation studies in the dietary assessment of children aged 12 to 36 months: a systematic literature review. J Nutr Sci. 2017;6:e16.

62. Ortega RM, Perez-Rodrigo C, Lopez-Sobaler AM. Dietary assessment methods: dietary records. Nutr Hosp. 2015;31(Suppl 3):38-45.

63. Seward K, Wolfenden L, Finch M, Wiggers J, Wyse R, Jones J, et al. Multistrategy childcare-based intervention to improve compliance with nutrition guidelines versus usual care in long day care services: a study protocol for a randomised controlled trial. BMJ Open. 2016;6(6):e010786.

64. Serdula MK, Alexander MP, Scanlon KS, Bowman BA. What are preschool children eating? A review of dietary assessment. Annu Rev Nutr. 2001;21(1):475-98.

65. Johnson RK. Dietary intake-how do we measure what people are really eating? Obes Res. 2002;10(S11):63S-8S.

66. van de Mortel TF. Faking it: social desirability response Bias in self-report research. Aust J Adv Nurs. 2008;25(4):40-8.

67. Thompson FE, Subar AF, Loria CM, Reedy JL, Baranowski T. Need for technological innovation in dietary assessment. J Am Diet Assoc. 2010; 110(1):48-51.

68. Martin CK, Nicklas T, Gunturk B, Correa JB, Allen HR, Champagne C. Measuring food intake with digital photography. J Hum Nutr Diet. 2014; 27(0 1):72-81.

69. Australian Children's Education \& Care Quality Authority. National RegisterWA 2016. Available from: http://www.acecqa.gov.au/national-registers/wa. Accessed May 2015.

70. Xyris Software. FoodWorks 9 professional [software]. Qld: High Gate Hill; 2018.

71. Environmental Health Australia. I'M ALERT 2016. Available from: https:// www.imalert.com.au/. Accessed 2015-2017.

72. Baxter J. Child care and early childhood education in Australia. In: Australian Institute of Family Studies, editor. Melbourne: Commonwealth of Australia; 2015.

73. Microsoft Office. Access. Microsoft; 2016.

74. IBM Corp. IBM SPSS statistics for windows. Version 250. Armonk: IBM Corp; 2018.

75. Eat for Health. Discretionary food and drink choices, : Australian government - National Health and Medical Research Council; 2017. Available from: https://www.eatforhealth.gov.au/food-essentials/ discretionary-food-and-drink-choices. [cited 201916 May]

76. National Health and Medical Research Council. What are nutrient reference values? Australia: Ministry of Health; 2018. Available from: https://www.nrv. gov.au/introduction. Accessed Jan 2018

\section{Publisher's Note}

Springer Nature remains neutral with regard to jurisdictional claims in published maps and institutional affiliations.

\section{Ready to submit your research? Choose BMC and benefit from:}

- fast, convenient online submission

- thorough peer review by experienced researchers in your field

- rapid publication on acceptance

- support for research data, including large and complex data types

- gold Open Access which fosters wider collaboration and increased citations

- maximum visibility for your research: over $100 \mathrm{M}$ website views per year

At $\mathrm{BMC}$, research is always in progress.

Learn more biomedcentral.com/submissions 